\title{
NODAL SETS OF LAPLACE EIGENFUNCTIONS: POLYNOMIAL UPPER ESTIMATES OF THE HAUSDORFF MEASURE.
}

\author{
ALEXANDER LOGUNOV
}

\begin{abstract}
Let $\mathbb{M}$ be a compact $C^{\infty}$-smooth Riemannian manifold of dimension $n, n \geq 3$, and let $\varphi_{\lambda}: \Delta_{M} \varphi_{\lambda}+\lambda \varphi_{\lambda}=0$ denote the Laplace eigenfunction on $\mathbb{M}$ corresponding to the eigenvalue $\lambda$. We show that

$$
H^{n-1}\left(\left\{\varphi_{\lambda}=0\right\}\right) \leq C \lambda^{\alpha},
$$

where $\alpha>1 / 2$ is a constant, which depends on $n$ only, and $C>0$ depends on $\mathbb{M}$. This result is a consequence of our study of zero sets of harmonic functions on $C^{\infty}$-smooth Riemannian manifolds. We develop a technique of propagation of smallness for solutions of elliptic PDE that allows us to obtain local bounds from above for the volume of the nodal sets in terms of the frequency and the doubling index.
\end{abstract}

\section{Preliminaries}

Yau conjectured that the Laplace eigenfunctions $\varphi_{\lambda}: \Delta \varphi_{\lambda}+\lambda \varphi_{\lambda}=0$ on a compact $C^{\infty}$-smooth Riemannain manifold $W$ of dimension $n$ (without boundary) satisfy

$$
c \lambda^{1 / 2} \leq H^{n-1}\left(\left\{\varphi_{\lambda}=0\right\}\right) \leq C \lambda^{1 / 2},
$$

where $H^{n-1}(\cdot)$ denotes the $(n-1)$ dimensional Hausdorf measure, positive constants $c, C$ depend on the Riemannian metric and on the manifold only. This conjecture was proved for real-analytic manifolds by Donnelly and Fefferman ([5]). For non-analytic manifolds the best-known upper estimate in dimension $n=2$ was $H^{1}\left(\left\{\varphi_{\lambda}=0\right\}\right) \leq C \lambda^{3 / 4}$ due to Donnelly and Fefferman ([6]), different proof for the same bound was given by Dong ([4]). Recently this bound was refined to $C \lambda^{3 / 4-\varepsilon}$ in [12].

In higher dimensions the estimate $H^{n-1}\left(\left\{\varphi_{\lambda}=0\right\}\right) \leq C \lambda^{C \sqrt{\lambda}}$ by Hardt and Simon ([9]) was the only known upper bound till now. We prove that

$$
H^{n-1}\left(\left\{\varphi_{\lambda}=0\right\}\right) \leq C \lambda^{\alpha}
$$

where $\alpha>1 / 2$ is a constant, which depends on $n$ only, $C$ depends on $M$.

This estimate will follow from an estimate (Theorem 6.1) for harmonic functions, which bounds the volume of the nodal set in terms of the frequency function (or the doubling index).

There is a standard trick that allows to pass from Laplace eigenfunctions to harmonic functions: one can add an extra variable $t$ and consider a 
function

$$
u(x, t)=\varphi(x) \exp (\sqrt{\lambda} t)
$$

which appears to be a harmonic function on the product manifold $W \times \mathbb{R}$.

Let $M$ be a $C^{\infty}$-smooth Riemannian manifold (non-compact and with no boundary), endowed with metric $g$. Consider a point $p \in M$ and a harmonic function $u$ (with respect to $g$ ) on $M$. By $B_{g}(p, r)$ we will denote a geodesic ball with center at point $p$ and radius $r$. Define $H(r)=\int_{\partial B_{g}(p, r)} u^{2} d S_{r}$, where $S_{r}$ is the surface measure on $\partial B_{g}(p, r)$ with respect to $g$. We will always assume that $r$ is smaller than the injectivity radius.

Definition. The frequency function of a harmonic function $u$ is defined by

$$
\beta(r):=\frac{r H^{\prime}(r)}{2 H(r)} .
$$

We remark that this definition is slightly different from the standard one, since we don't normalize $H(r)$ by the surface measure $\left|S_{r}\right|$. See [10] for a friendly introduction to frequency and also [8], [1] for applications to nodal sets. In dimension two understanding of nodal sets of harmonic functions is better due to complex analysis techniques and topological reasons, see [16].

We will work only on a bounded subset of $M$ : fix a point $O$ on $M$ and assume hereafter that $B_{g}(p, r) \subset B_{g}(O, 1)$.

The frequency is almost monotonic in the following sense (see Remark (3) to Theorem 2.2 in [14]):

Lemma 1.1. For any $\varepsilon>0$ there exists $R_{0}=R_{0}(\varepsilon, M, g, O)$ such that

$$
\beta\left(r_{1}\right) \leq(1+\varepsilon) \beta\left(r_{2}\right)
$$

for any $r_{1}, r_{2}$ : $0<r_{1}<r_{2}<R_{0}$.

One can estimate the growth of $H(r)$ in terms of the frequency in view of the integral formula:

$$
\frac{H\left(r_{2}\right)}{H\left(r_{1}\right)}=\exp \left(2 \int_{r_{1}}^{r_{2}} \beta(r) d \log r\right) .
$$

Corollary 1.2. $\left(\frac{r_{2}}{r_{1}}\right)^{2 \beta\left(r_{1}\right) /(1+\varepsilon)} \leq \frac{H\left(r_{2}\right)}{H\left(r_{1}\right)} \leq\left(\frac{r_{2}}{r_{1}}\right)^{2 \beta\left(r_{2}\right)(1+\varepsilon)}$.

Sometimes we will specify the center of the ball and our choice of the function $u$ and write $\beta(p, r)$ and $H(p, r)$ or $\beta_{u}(p, r)$ and $H_{u}(p, r)$ in place of $\beta(r)$ and $H(r)$.

We need a standard elliptic estimate that compares $L^{\infty}$ and $L^{2}$ norms of harmonic functions on concentric geodesic spheres: for any $\varepsilon \in(0,1)$ there exists a constant $C_{1}=C_{1}(\varepsilon, M, g, O)>0$ such that

$$
\sup _{\partial B_{g}(p, r)}|u|^{2} \leq C_{1} \frac{H(r(1+\varepsilon))}{r^{n-1}}
$$

for $r \leq R_{0}$, where $R_{0}=R_{0}(M, g, O)>0$. 
The reverse estimate holds for arbitrary continuous functions on $M$ :

$$
H(r) \leq C_{2}(M, g, O) r^{n-1} \sup _{\partial B_{g}(p, r)}|u|^{2},
$$

where $C_{2}(M, g, O)$ is a positive constant such that the whole surface measure of a geodesic sphere $\left|S_{r}\right| \leq r^{n-1} C_{2}(M, g), r \leq R_{0}$.

Let us consider normal coordinates in a geodesic ball $B_{g}(O, R)$, where $R$ is a sufficiently small number. In these coordinates we will treat the Laplace operator as an elliptic operator in a fixed domain in $\mathbb{R}^{n}$, say, a cube $Q$. We will identify $O$ with the origin and denote the ordinary Euclidean distance by $d(x, y)$ and the Riemannian distance by $d_{g}(x, y)$. Let $\varepsilon>0$ be a small number. We will assume hereafter that

$$
\frac{d_{g}(x, y)}{d(x, y)} \in(1-\varepsilon, 1+\varepsilon)
$$

for points $x, y$ in $B_{g}\left(O, R_{0}\right): x \neq y$, where $R_{0}=R_{0}(\varepsilon, M, g, O)>0$. The existence of such $R_{0}$ for any $\varepsilon$ is provided by the choice of the normal coordinates.

For the purposes of the paper it will be more convenient to work with a notion similar to the frequency: so-called doubling index, which deals with $L^{\infty}$ norms in place of $L^{2}$ and Euclidean balls in place of geodesic balls. For a given ball $B$ (ball in standard Euclidean metric) define the doubling index $N(B)$ by $2^{N(B)}=\frac{\sup ^{2 B}|u|}{\sup _{B}|u|}$. Given a positive number $r$ we denote by $r B$ the homothety image of $B$ with coefficient $r$ such that $r B$ and $B$ have the same center. If $B$ is an Euclidean ball in $\mathbb{R}^{n}$ with center at $x$ and radius $r$, then $N(x, r)$ will denote the doubling index for this ball.

We will use the estimates of growth of harmonic functions in terms of the doubling index.

Lemma 1.3. For any $\varepsilon \in(0,1)$ there exist $C=C(\varepsilon, M, g, O)>0$ and $R=R(\varepsilon, M, g, O)>0$ such that

$$
t^{N(x, \rho)(1-\varepsilon)-C} \leq \frac{\sup _{B(x, t \rho)}|u|}{\sup _{B(x, \rho)}|u|} \leq t^{N(x, t \rho)(1+\varepsilon)+C}
$$

for any $x \in M$ and numbers $\rho>0, t>2$ satisfying $B(x, t \rho) \subset B(O, R)$ (and for any harmonic function $u)$. Furthermore, there exists $N_{0}=N_{0}(\varepsilon, M, g)$ such that if additionally $N(x, \rho)>N_{0}$, then

$$
t^{N(x, \rho)(1-\varepsilon)} \leq \frac{\sup _{B(x, t \rho)}|u|}{\sup _{B(x, \rho)}|u|} .
$$

The estimates (5), (6) are corollaries from almost monotonicity of the frequency (1) and standard elliptic estimates. For the convenience of the reader we deduce them in Lemma 7.2 and Lemma 7.3. 
We will show in Theorem 6.1 that there exist $r=r(M)>0$ and $\alpha=$ $\alpha(n)>1$ such that the following inequality holds:

$$
H^{n-1}(\{u=0\} \cap B(O, r)) \leq C(N(O, K r))^{\alpha},
$$

where $K=K(n) \geq 2$ and $C=C(M)$.

Note that for real analytic manifolds one can replace $\alpha$ by 1 in the estimate above, using complex analysis techniques (holomorhpic extension of a harmonic function to an open set in $\mathbb{C}^{n}$ and Jensen's formula on one dimensional sections), see 8 .

We remark that only few properties of $H^{n-1}$ are used in the proof: subadditivity and the rescaling property. So there is a chance that the methods of this paper might be applied to other characteristics of nodal sets.

We outline the question we are trying to investigate in this paper: Is the frequency additive in some sense?

Some partial positive answers are obtained in the simplex lemma and in the hyperplane lemma, which are combined to get the polynomial upper bounds for the volume of the nodal sets in terms of the frequency (or the doubling index).

Acknowledgments. This work was started in collaboration with Eugenia Malinnikova who suggested to apply the combinatorial approach to nodal sets of Laplace eigenfunctions. Her role in this work is no less than the author's one. Unfortunately, she refused to be a coauthor of this paper. On various stages of this work I discussed it with Lev Buhovsky and Mikhail Sodin. Eugenia, Lev and Mikhail also read the first draft of this paper and made many suggestions and comments. I thank all of them.

This work was started while the author was visiting NTNU, continued at the Chebyshev Laboratory (SPBSU) and finished at TAU. The final version of the paper was completed at the Institute for Advanced Study. I am grateful to these institutions for their hospitatility and for great working conditions.

The author was supported in part by ERC Advanced Grant 692616 and ISF Grants 1380/13, 382/15 and by a Schmidt Fellowship at the Institute for Advanced Study.

\section{SimpleX LEMmA}

Let $x_{1}, \ldots, x_{n+1}$ be vertices of a simplex $S$ in $\mathbb{R}^{n}$. The $\operatorname{symbol} \operatorname{diam}(S)$ will denote the diameter of $S$ and by $w i d t h(S)$ we will denote the width of $S$, i.e. the minimum distance between a pair of parallel hyperplanes such that $S$ is contained between them. Define the relative width of $S$ : $w(S)=\operatorname{width}(S) / \operatorname{diam}(S)$. Let $a>0$ and assume that $w(S)>a$. In particular we assume that $x_{1}, \ldots, x_{n+1}$ do not lie on the same hyperplane. For the purposes of the paper there will be sufficient a particular choice of $a$, which depends on the dimension $n$ only, the choice will be specified in Section 5. Denote by $x_{0}$ the barycenter of $S$. 
We will use an Euclidean geometry lemma: there exist $c_{1}>0, K \geq 2 / a$ depending on $a, n$ only such that if $\rho=K \operatorname{diam}(S)$, then $B\left(x_{0}, \rho\left(1+c_{1}\right)\right) \subset$ $\cup_{i=1}^{n+1} B\left(x_{i}, \rho\right)$.

We remark that if the simplex is very degenerate ( $a$ is small), then $c_{1}$ has to be small and the number $K$ has to be big:

$$
c_{1} \rightarrow 0, K \rightarrow+\infty \text { as } a \rightarrow 0 \text {. }
$$

Lemma 2.1. Let $B_{i}$ be balls with centers at $x_{i}$ and radii not greater than $\frac{K}{2} \operatorname{diam}(S), i=1, \ldots, n+1$, where $K=K(a, n)$ is from the Euclidean geometry lemma. There exist positive numbers $c=c(a, n), C=C(a, n) \geq$ $K, r=r(M, g, O, a), N_{0}=N_{0}(M, g, O, a)$ such that if $S \subset B(O, r)$ and if $N\left(B_{i}\right)>N$ for each $x_{i}, i=1 \ldots n+1$, where $N$ is a number greater than $N_{0}$, then $N\left(x_{0}, C \operatorname{diam}(S)\right)>N(1+c)$.

Proof. In view of almost monotonicity of the doubling index (5) we will assume that all $B_{i}$ have the same radius $\rho=\operatorname{Kdiam}(S)$.

Let $M$ be the supremum of $|u|$ over the union of $B\left(x_{i}, \rho\right)$, then $|u|$ is not greater than $M$ in $B\left(x_{0}, \rho\left(1+c_{1}\right)\right)$ and $\sup _{B\left(x_{i}, \rho\right)}|u|=M$ for some $i$. Let $t>2$ and $\varepsilon>0$, these parameters will be specified later. Assume that (6) holds for $B\left(x_{i}, \rho t\right)$, then $\sup _{B\left(x_{i}, \rho t\right)}|u| \geq M t^{N(1-\varepsilon)}$.

We need a metric geometry fact, which follows from the triangle inequality: there exists $\delta=\delta(t) \in(0,1)$ such that $B\left(x_{i}, \rho t\right) \subset B\left(x_{0}, \rho t(1+\delta)\right)$ and $\delta(t) \rightarrow 0$ as $t \rightarrow+\infty$.

Let $\widetilde{N}$ be the doubling index for $B\left(x_{0}, \rho t(1+\delta)\right)$. Suppose (5) holds for the pair of balls $B\left(x_{0}, \rho t(1+\delta)\right)$ and $B\left(x_{0}, \rho\left(1+c_{1}\right)\right)$, then

$$
\left[\frac{t(1+\delta)}{1+c_{1}}\right]^{\tilde{N}(1+\varepsilon)+C} \geq \frac{\sup _{B\left(x_{0}, \rho t(1+\delta)\right)}|u|}{\sup _{B\left(x_{0}, \rho\left(1+c_{1}\right)\right)}|u|} \geq \frac{\sup _{B\left(x_{i}, \rho t\right)}|u|}{\sup _{B\left(x_{0}, \rho\left(1+c_{1}\right)\right)}|u|} \geq \frac{M t^{N(1-\varepsilon)}}{M}=t^{N(1-\varepsilon)} .
$$

Hence

$$
\left[\frac{t(1+\delta)}{1+c_{1}}\right]^{\widetilde{N}(1+\varepsilon)+C} \geq t^{N(1-\varepsilon)}
$$

Now, we specify our choice of parameters. We first choose $t>2$ so that $\delta(t)<c_{1} / 2$, then

$$
\frac{t(1+\delta)}{1+c_{1}} \leq t^{1-c_{2}}
$$

for some $c_{2}=c_{2}\left(t, c_{1}\right) \in(0,1)$. Second, we choose $\varepsilon=\varepsilon\left(c_{2}\right)>0$ and $c=c\left(c_{2}\right)>0$ such that

$$
\frac{1-\varepsilon}{(1+\varepsilon)\left(1-c_{2}\right)}>1+2 c
$$

Third, we choose $R=R(\varepsilon, M, g, O)>0$ and $N_{0}=N_{0}(\varepsilon, M, g, O)$ such that Lemma 1.3 holds for these parameters and put $r:=R /(10 K t)$. This choice 
of $r$ provides (5) for the pair of balls $B\left(x_{0}, \rho t(1+\delta)\right)$ and $B\left(x_{0}, \rho\left(1+c_{1}\right)\right)$ and (6) for $B\left(x_{i}, \rho t\right)$. Hence the inequality (7) holds and (8) gives

$$
t^{\left(1-c_{2}\right)(\widetilde{N}(1+\varepsilon)+C)} \geq t^{N(1-\varepsilon)} .
$$

We therefore have

$$
\widetilde{N} \geq N \frac{(1-\varepsilon)}{(1+\varepsilon)\left(1-c_{2}\right)}-C_{1} \geq N(1+2 c)-C_{1} \geq N(1+c)+\left(c N_{0}-C_{1}\right) .
$$

We can also ask $N_{0}$ to be big enough so that $c N_{0}-C_{1}>0$. Thus

$$
\widetilde{N}>N(1+c) \text {. }
$$

\section{Propagation of smallness of the Cauchy data}

If one considers a smooth Riemannian metric $g$ in a unit cube $Q$ in $\mathbb{R}^{n}$, then any harmonic function $u$ (with respect to $g$ ) satisfies $L u=0$, where $L$ is a uniformly elliptic (in a slightly smaller cube) operator of second order in the divergence form with smooth coefficients. Consider a cube $q \subset \frac{1}{2} Q$ with side $r$ and let $F$ be a face of $q$. In this section we formulate a result that we will refer to as the propagation of smallness of the Cauchy data for elliptic PDE. See Lemma 4.3 in [11] and Theorem 1.7 in [1] for the proof of the result below, which we bring not in full generality but in a convenient way for our purposes.

Suppose that $|u| \leq 1$ in $q$. There exist $C>0$ and $\alpha \in(0,1)$, depending on $L$ only such that if $|u| \leq \varepsilon$ on $F$ and $|\nabla u| \leq \frac{\varepsilon}{r}$ on $F, \varepsilon<1$, then

$$
\sup _{\frac{1}{2} q}|u| \leq C \varepsilon^{\alpha} \text {. }
$$

Remark 3.1. We will apply propagation of smallness of the Cauchy data in the case when the coefficients of the operator $L$ are sufficiently close in the $L^{\infty}$ norm to the coefficients of the standard Laplace operator $\Delta$ in $B\left(O, R_{0}\right)$ and the derivatives of coefficients $L$ are sufficiently small. Under these assumptions $\alpha$ can be chosen to depend only on $n$, see Theorem 1.7 in [1].

\section{Hyperplane Lemma}

Given a cube $Q$, we will denote $\sup _{x \in Q, r \in(0, \operatorname{diam}(Q))} N(x, r)$ by $N(Q)$ and call it the doubling index of $Q$. This definition is different than a doubling index for balls but more convenient in the following sense. If a cube $q$ is contained in a cube $Q$, then $N(q) \leq N(Q)$. Furthermore if a cube $q$ is covered by cubes $Q_{i}$ with $\operatorname{diam}\left(Q_{i}\right) \geq \operatorname{diam}(q)$, then $N\left(Q_{i}\right) \geq N(q)$ for some $Q_{i}$.

Lemma 4.1. Let $Q$ be a cube $[-R, R]^{n}$ in $\mathbb{R}^{n}$. Divide $Q$ into $(2 A+1)^{n}$ equal subcubes $q_{i}$ with side-length $\frac{2 R}{2 A+1}$. Consider the cubes $q_{i, 0}$ that have nonempty intersection with the hyperplane $x_{n}=0$. Suppose that for each $q_{i, 0}$ 
there exists $x_{i} \in q_{i, 0}$ and $r_{i}<10$ diam $\left(q_{i, 0}\right)$ such that $N\left(x_{i}, r_{i}\right)>N$, where $N$ is a given positive number. Then there exist $A_{0}=A_{0}(n), R_{0}=R_{0}(M, g, O)$, $N_{0}=N_{0}(M, g, O)$ such that if $A>A_{0}, N>N_{0}, R<R_{0}$ then $N(Q)>2 N$.

Proof. We will ask $R_{0}$ to be small enough so that Lemma 1.3 holds with $\varepsilon=1 / 2$ and $10 n \cdot R_{0}$ in place of $R$ in Lemma 1.3. Also we may assume that coefficients of $L$ are close to the coefficients of the standard Laplacian in $C^{1}\left(B\left(O, 10 n \cdot R_{0}\right)\right)$ to be able to use (10). We have described our choice of $R_{0}$.

For the sake of simplicity we will assume that $R=1 / 2$ and $R_{0} \geq 1 / 2$. The general case follows by changing the scale in the argument below.

Let $B$ be the unit ball $B(O, 1)$ and let $M$ be the supremum of $u$ over $\frac{1}{8} B$. For each $x_{i}$ in $\frac{1}{16} B$ the ball $B\left(x_{i}, 1 / 32\right)$ is contained in $1 / 8 B$. Hence sup $|u| \leq M$. Using $N\left(x_{i}, r_{i}\right)>N$ and (5) with $\varepsilon=1 / 2$ we get $B\left(x_{i}, 1 / 32\right)$

$$
\sup _{2 q_{i, 0}}|u| \leq \sup _{B\left(x_{i}, \frac{4 \sqrt{n}}{2 A+1}\right)}|u| \leq C \sup _{B\left(x_{i}, 1 / 32\right)}|u|\left(\frac{128 \sqrt{n}}{2 A+1}\right)^{\frac{N}{2}} \leq M 2^{-c N \log A},
$$

where $c=c(n)>0$. In the last inequality we assumed that $A>A_{0}(n)$ and $N$ is sufficiently large .

By a standard elliptic estimate

$$
\sup _{q_{i, 0}}|\nabla u| \leq C A \sup _{2 q_{i, 0}}|u| \leq C A M 2^{-c N \log A} \leq M 2^{-c_{1}(n) N \log A} .
$$

Thus $|u|$ and $|\nabla u|$ are bounded by $M 2^{-c_{1} N \log A}$ on $\frac{1}{8} B \cap\left\{x_{n}=0\right\}$.

Let $q$ be a cube with side $\frac{1}{16 \sqrt{n}}$ in the halfspace $\left\{x_{n}>0\right\}$ such that $q \subset \frac{1}{8} B$ and

$$
\frac{1}{32 \sqrt{n}} B \cap\left\{x_{n}=0\right\} \subset \partial q \cap\left\{x_{n}=0\right\}
$$

In other words, $q$ has a face $F$ on the hyperplane $\left\{x_{n}=0\right\}$. Let $p$ be the center of $q$, then $B\left(p, \frac{1}{32 \sqrt{n}}\right) \subset q$.

Consider the function $v=\frac{u}{M}$, which absolute value is not greater than 1 in $q$. The Cauchy data of $v$ is small on $F:|v|$ and $|\nabla v|$ are smaller than $2^{-c_{1} N \log A}$. Denote $2^{-c_{1} N \log A}$ by $\varepsilon$. Applying propagation of smallness for the Cauchy data, we obtain $\sup |v| \leq \varepsilon^{\alpha}$. In terms of $u$ we have $\sup |u| \leq$ $M \varepsilon^{\alpha}=M 2^{-\alpha c_{1} N \log A}$.

$$
\frac{1}{2} q
$$

The ball $B\left(p, \frac{1}{64 \sqrt{n}}\right)$ is contained in $\frac{1}{2} q$ and therefore

$$
\sup _{B\left(p, \frac{1}{64 \sqrt{n}}\right)}|u| \leq M 2^{-\frac{\alpha c N}{2} \log A} .
$$


However $\sup _{B(p, 1 / 2)}|u| \geq M$ since $\frac{1}{8} B \subset B(p, 1 / 2)$. Hence

$$
\frac{\sup _{B(p, 1 / 2)}|u|}{\sup _{B\left(p, \frac{1}{64 \sqrt{n}}\right)}|u|} \geq 2^{\alpha c_{1} N \log A} .
$$

Denote by $\tilde{N}$ the doubling index for $B(p, 1 / 2)$. By (5) with $\varepsilon=1 / 2$ we have

$$
\frac{\sup _{B(p, 1 / 2)}|u|}{\sup _{B\left(p, \frac{1}{64 \sqrt{n}}\right)}|u|} \leq(64 \sqrt{n})^{\tilde{N} / 2} \text {. }
$$

Hence $\tilde{N} \geq c_{2} N \log A$ for some $c_{2}=c_{2}(n)>0$, and $\tilde{N} \geq 2 N$ for $A$ big enough.

Corollary 4.2. Let $Q$ be a cube $[-R, R]^{n}$ in $\mathbb{R}^{n}$ and $N(Q)$ is not greater than a number $N$. For any $\varepsilon>0$ there exists an odd positive integer $A_{1}=$ $A_{1}(n, \varepsilon)$ such that the following holds. Let us divide $Q$ into $A_{1}^{n}$ smaller equal subcubes $q_{i}$ and consider the cubes $q_{i, 0}$ that have non-empty intersection with the hyperplane $x_{n}=0$. If $N>N_{0}(M, g, O), R<R_{0}(M, g, O)$, then the number of subcubes $q_{i, 0}$ that have doubling index greater than $N / 2$ is less than $\varepsilon A_{1}^{n-1}$.

Proof. According to Lemma 4.1 we can choose an integer $A_{0}$ and $N_{0}>0$, assume $N>N_{0}$, partition $Q$ into $\left(2 A_{0}+1\right)^{n}$ equal subcubes, and then at least one subcube with non-empty intersection with $\left\{x_{n}=0\right\}$ has doubling index smaller than $N / 2$.

Now, let us partition $Q$ into $\left(2 A_{0}+1\right)^{k n}$ equal subcubes $q_{i}$ and denote by $M_{k}$ the number of subcubes with non-empty intersection with $\left\{x_{n}=0\right\}$ and doubling index greater than $N / 2$. If a cube $q_{i}$ has doubling index smaller than $N / 2$, then any its subcube also does.

It is not important in the proof of Lemma 4.1 that $Q$ is a cube with center at the origin, the same argument shows that if we divide a cube $q_{i}$, which has non-zero intersection with $\left\{x_{n}=0\right\}$, into $\left(2 A_{0}+1\right)^{n}$ equal subcubes, then at least one subcube with non-empty intersection with $\left\{x_{n}=0\right\}$ has doubling index smaller than $N / 2$. This observation gives $M_{k+1} \leq M_{k}\left(\left(2 A_{0}+1\right)^{n-1}-\right.$ $1)$.

Thus $M_{k} \leq\left(1-\frac{1}{\left(2 A_{0}+1\right)^{n-1}}\right)^{k}\left(2 A_{0}+1\right)^{k(n-1)}$. Choosing $k$ so that $(1-$ $\left.\frac{1}{\left(2 A_{0}+1\right)^{n-1}}\right)^{k} \leq \varepsilon$, we finish the proof.

Remark 4.3. The same argument shows that in Lemma 4.1 and in Corollary 4.2 one can replace $Q$ by any its homothety-rotation-shift copy $Q_{r} \subset$ $B\left(O, R_{0}\right), r \in(0,1), R_{0}=R_{0}(M, g, O)$ and replace the hyperplane $\left\{x_{n}=0\right\}$ by a hyperplane that contains the center of $Q_{r}$ and is parallel to one of its faces, Lemma 4.1 and Corollary 4.2 will remain true with $A_{1}, A_{0}$ and $N_{0}$ independent of $r$. 


\section{Number of CUbes With BIG DOUbling INDEX}

In this section we follow notation from Sections 1 and 2, The next theorem seems to be a useful tool in nodal geometry. We will apply it later to obtain upper estimates of the volume of the nodal sets in terms of the doubling index.

Theorem 5.1. There exist constants $c>0$, an integer $A$ depending on the dimension $d$ only and positive numbers $N_{0}=N_{0}(M, g, O), r=r(M, g, O)$ such that for any cube $Q \subset B(O, r)$ the following holds: if we partition $Q$ into $A^{n}$ equal subcubes, then the number of subcubes with doubling index greater than $\max \left(N(Q) /(1+c), N_{0}\right)$ is less than $\frac{1}{2} A^{n-1}$.

Proof. Let us fix a small $\varepsilon>0$, which will be specified later, and choose $A_{1}=A_{1}(\varepsilon, n)$ such that Corollary 4.2 holds for this $\varepsilon$ and $A_{1}=2 A_{0}+1$ as well as the remark after Corollary 4.2 . Let us subsequently divide $Q$ into equal subcubes so that at $j$-th division step $Q$ is partitioned into $\left(2 A_{0}+\right.$ $1)^{n j}$ equal subcubes $Q_{i_{1}, i_{2}, \ldots, i_{j}}, i_{1}, i_{2} \ldots, i_{j} \in\left\{1,2, \ldots,\left(2 A_{0}+1\right)^{n}\right\}$, so that $Q_{i_{1}, i_{2}, \ldots, i_{j}} \subset Q_{i_{1}, i_{2}, \ldots, i_{j-1}}$. Let the parameter $c>0$. We will say that the cube $Q_{i_{1}, i_{2}, \ldots, i_{j}}$ is bad if $N\left(Q_{i_{1}, i_{2}, \ldots, i_{j}}\right)>N(Q) /(1+c)$ and good otherwise.

Fix a cube $Q_{i_{1}, i_{2}, \ldots, i_{j}}=: q$, we are interested in the number of its bad subcubes $Q_{i_{1}, i_{2}, \ldots, i_{j+1}}=: q_{i_{j+1}}$. For the sake of convenience we will omit index $j+1$ and write $q_{i}$ in place of $q_{i_{j+1}}$. We will prove the following lemma.

Lemma 5.2. If $\varepsilon, c$ are sufficiently small, and $j>j_{0}$, where $j_{0}=j_{0}(\varepsilon, c)$, then $\#\left\{i: N\left(q_{i}\right)>N(Q) /(1+c)\right\} \leq \frac{1}{2}\left(2 A_{0}+1\right)^{n-1}$

Let $F$ be the set of all points $x$ in $q$ such that there exists $r \in\left(0, \operatorname{diam}\left(q_{i}\right)\right]$ such that $N(x, r)>N(Q) /(1+c)$. If a closed cube $q_{i}$ is bad, then it contains at least one point from $F$. We use the notation $\widetilde{w}(F):=\frac{\operatorname{width}(F)}{\operatorname{diam}(q)}$ for the relative width of $F$ in $q$. We will prove Lemma 5.2 after the following lemma.

Lemma 5.3. For any $w_{0}>0$ there exist a positive integer $j_{0}$ and a constant $c_{0}>0$ such that if $j>j_{0}, c<c_{0}$, then $\widetilde{w}(F)<w_{0}$.

To prove this lemma we need an Euclidean geometry fact: for any set of points $F$ in $q$ with non-zero $\widetilde{w}(F)$ there exists $a=a(\widetilde{w}(F), n)>0$ and $a$ simplex $S \subset F$ such that $w(S)>a$ and $\operatorname{diam}(S)>a \cdot \operatorname{diam}(q)$.

For each vertex $x_{k}$ of $S$ there is a ball $B\left(x_{k}, r_{k}\right)$ with $N\left(x_{k}, r_{k}\right) \geq N /(1+c)$ and $r_{k} \leq \operatorname{diam}(q) \leq \frac{1}{a} \operatorname{diam}(S)$. We can apply Lemma 2.1 for the simplex $S$. Then $N\left(x_{0}, C_{0} \operatorname{diam}(S)\right)>\left(1+c_{0}\right) N /(1+c)$, where $x_{0}$ is a barycenter of $S$ and $c_{0}, C_{0}$ are positive constants depending on $a$ (and $n$ ) only and therefore on $\widetilde{w}(F)$ only (and $n$ ). If $c_{0}>c$ and $C_{0} \operatorname{diam}(S) \leq \operatorname{diam}(Q)$ that means a contradiction with $N(Q) \leq N$. This is why we require $j$ to be big enough: $\operatorname{diam}(S) \leq \operatorname{diam}(q) \leq \frac{\operatorname{diam}(Q)}{\left(2 A_{0}+1\right)^{j}} \leq \operatorname{diam}(Q) / 3^{j}$ and it is sufficient to take $j$ such that $3^{j}>C_{0}$.

Now, Lemma 5.3 is proved and we can think that $\widetilde{w}(F)$ is smaller than a fixed number $w_{0}=\frac{1}{2 A_{0}+1}$ and proceed to prove Lemma 5.2. There exists 
a hyperplane $P$ such that its $w_{0} \cdot \operatorname{diam}(q)$ neighborhood contains all $F$. Furthermore, we can find a biggier cube $\widetilde{q}$ with one face parallel to $P$ such that the center of $\widetilde{q}$ is in $P \cap q$ and $\operatorname{diam}(\widetilde{q})=10 \sqrt{n} \cdot \operatorname{diam}(q)$. Automatically $\widetilde{q}$ contains $q$. Divide $\widetilde{q}$ into $\left(2 A_{0}+1\right)^{n}$ equal subcubes $\widetilde{q}_{i}$. We will denote by $\widetilde{q}_{i, 0}$ such subcubes that have non-zero intersection with $P$. Since $w_{0} \leq \frac{1}{2 A_{0}+1}$, each bad cube $q_{i}$ is contained in a $\frac{2 \sqrt{n} \cdot \operatorname{diam}(q)}{2 A_{0}+1}$ neighborhood of $P$ and each bad cube $q_{i}$ is covered by a finite number (which depends on $n$ only) of $\widetilde{q}_{i, 0}$. Therefore the number of bad cubes $q_{i}$ is less than the number of bad cubes $\widetilde{q}_{i, 0}$ times some constant depending on dimension $n$ only.

Now, assume the contrary to Lemma 5.2, Suppose that the number of bad $q_{i}$ is greater than $\frac{1}{2}\left(2 A_{0}+1\right)^{n-1}$, then the number of bad cubes $\widetilde{q}_{i, 0}$ is at least $\frac{1}{C}\left(2 A_{0}+1\right)^{n-1}$, where $C=C(n)>0$.

Finally, we choose $\varepsilon$, which didn't play a role till now: $\varepsilon$ is any number in $\left(0, \frac{1}{2 C}\right)$. Recall that $A_{0}=A_{0}(\varepsilon)$ is such that Corollary 4.2 holds for $A_{1}=2 A_{0}+1$ and this $\varepsilon$ as well as the remark after Corollary 4.2. Since the number of bad $\widetilde{q}_{i, 0}$ is greater than $\varepsilon\left(2 A_{0}+1\right)^{n-1}$ we have $N(\widetilde{q}) \geq 2 N /(1+c)$. Without loss of generality we assume that $c<1 / 10$, then there exists a point $\widetilde{p} \in \widetilde{q}$ such that $N(\widetilde{p}, \operatorname{diam}(\widetilde{q})) \geq \frac{3}{2} N$. The last observation looks to be inconsistent with $N(Q) \leq N$, however $\widetilde{q}$ is not necessarily contained in $Q$ and the contradiction is not immediate. This obstacle is easy to overcome. Consider any point $p \in q \subset Q$. There exists a large $C_{1}=C_{1}(n)$ such that $N\left(p, C_{1} \operatorname{diam}(\widetilde{q})\right) \geq(1-1 / 100) N(\widetilde{p}, \operatorname{diam}(\widetilde{q}))$ (see Lemma 7.4). Thus there is a contradiction with $N(Q) \leq N$ since $N\left(p, C_{1} \operatorname{diam}(\widetilde{q})\right)>N$ and $C_{1} \operatorname{diam}(\widetilde{q}) \leq \operatorname{diam}(Q)$ if $j$ is big enough. The proof of Lemma 5.2 is completed. Now, it is a straightforward matter to prove Theorem 5.1.

Denote by $K_{j}$ the number of bad cubes on $j$-th step. If $Q_{i_{1}, i_{2}, \ldots, i_{j}}$ is good, then any its subcube is also good by the definition of doubling index for cubes. If $Q_{i_{1}, i_{2}, \ldots, i_{j}}=: q$ is bad, then by Lemma 5.2 the number of bad subscubes $Q_{i_{1}, i_{2}, \ldots, i_{j+1}}$ in $q$ is less than $\frac{1}{2}\left(2 A_{0}+1\right)^{n-1}$. Hence $K_{j+1} \leq$ $\frac{1}{2}\left(2 A_{0}+1\right)^{n-1} K_{j}$ for $j>j_{0}$. We can define $A=\left(2 A_{0}+1\right)^{j}$ and see that $K_{j} \leq K_{j_{0}} \frac{1}{2^{j-j_{0}}}\left(2 A_{0}+1\right)^{(n-1)\left(j-j_{0}\right)} \leq \frac{1}{2} A^{n-1}$ for $j$ big enough.

\section{UPPER ESTIMATES OF THE VOLUME OF THE NODAL SET.}

Theorem 6.1. There exist positive numbers $r=r(M, g, O), C=C(M, g, O)$ and $\alpha=\alpha(n)$ such that for any harmonic function $u$ on $M$ and any cube $Q \subset B(O, r)$

$$
H^{n-1}(\{u=0\} \cap Q) \leq \operatorname{Cdiam}^{n-1}(Q) N_{u}^{\alpha}(Q),
$$

where $N_{u}(Q)$ is the doubling index of $Q$ for the function $u$. 
Proof. Choose $r$ so that Theorem 5.1 holds with this $r$ and some $c=c(n)$, $A=A(n)$. Now, define the function

$$
F(N):=\sup \frac{H^{n-1}(\{u=0\} \cap Q)}{\operatorname{diam}^{n-1}(Q)},
$$

where the supremum is taken over the set of harmonic functions $u$ on $M$, which we denote by $\operatorname{Harm}(M)$, and cubes $Q$ within $B(O, r)$ such that $N_{u}(Q) \leq N$. The estimate (11) is equivalent to

$$
F(N) \leq C N^{\alpha} \text {. }
$$

We note that if $u$ changes a sign in $Q$, then $N_{u}(Q) \geq 1$, since $\lim _{t \rightarrow+0} N(x, t)$ is equal to the vanishing order of $u$ at $x$. Due to the Hardt-Simon exponential bounds we know $F(N)<+\infty$ for each positive $N$.

We will call $N>0$ bad if

$$
F(N)>4 A \cdot F(N /(1+c)) .
$$

Our goal is to show that the set of bad $N$ is bounded. In view of monotonicity of $F$ it would imply (12) immediately, where the constant $\alpha$ depends on $A$ and $c$ only and therefore only on the dimension $n$.

Consider a bad $N$ and a function $u$ with a cube $Q$ such that $F(N)$ is almost attained for them:

$$
\frac{H^{n-1}(\{u=0\} \cap Q)}{\operatorname{diam}^{n-1}(Q)}>\frac{3}{4} F(N)
$$

while $N_{u}(Q) \leq N$. Divide $Q$ into $A^{n}$ equal subcubes $Q_{i}, i=1, \ldots, A^{n}$. Divide $Q_{i}$ into two groups $G_{1}:=\left\{Q_{i}: N /(1+c)<N\left(Q_{i}\right) \leq N\right\}$ and $G_{2}:=\left\{Q_{i}: N\left(Q_{i}\right) \leq N /(1+c)\right\}$. By Theorem 5.1 we know that the number of cubes in $G_{1}$ satisfies $\left|G_{1}\right| \leq \frac{1}{2} A^{n-1}$ if $N>N_{0}(M, g)$. Note that

$$
\begin{gathered}
H^{n-1}(\{u=0\} \cap Q) \leq \sum_{Q_{i} \in G_{1}} H^{n-1}\left(\{u=0\} \cap Q_{i}\right)+\sum_{Q_{i} \in G_{2}} H^{n-1}\left(\{u=0\} \cap Q_{i}\right) \\
\leq\left|G_{1}\right| F(N) \frac{\operatorname{diam}^{n-1}(Q)}{A^{n-1}}+\left|G_{2}\right| F(N /(1+c)) \frac{\operatorname{diam}^{n-1}(Q)}{A^{n-1}}=I+I I .
\end{gathered}
$$

Since $\left|G_{1}\right| \leq \frac{1}{2} A^{n-1}$ we can estimate $I \leq \frac{1}{2} F(N) \operatorname{diam}^{n-1}(Q)$. Using that $N$ is bad, we have $I I \leq\left|G_{2}\right| \frac{F(N)}{4 A} \frac{\operatorname{diam}^{n-1}(Q)}{A^{n-1}}$ and $\left|G_{2}\right| \leq A^{n}$, hence $I I \leq$ $\frac{1}{4} F(N) \operatorname{diam}^{n-1}(Q)$. Finally, $H^{n-1}(\{u=0\} \cap Q) \leq \frac{3}{4} F(N) \operatorname{diam}^{n-1}(Q)$ and the last inequality contradicts to (14). Thus we had shown that the set of bad $N$ is bounded by some $N_{0}=N_{0}(M, g)$.

Theorem 6.2. Let $(W, g)$ be a compact $C^{\infty}$-smooth Riemannian manifold without boundary. For a Laplace eigenfunction $\varphi$ on $W$ with $\Delta \varphi+\lambda \varphi=0$ define its nodal set $Z_{\varphi}:=\{\varphi=0\}$. There exist $C=C(W, g)$ and $\alpha$, depending only on the dimension $n$ of $W$, such that

$$
H^{n-1}\left(Z_{\varphi}\right) \leq C \lambda^{\alpha} .
$$


Proof. We will use a standard trick that allows to pass from Laplace eigenfunctions to harmonic functions by adding an extra variable. Consider a product manifold $M=W \times \mathbb{R}$, where one can define a harmonic function $u$ by

$$
u(x, t)=\varphi(x) e^{\sqrt{\lambda} \cdot t}, x \in W, t \in \mathbb{R} .
$$

The Donnelly-Fefferman doubling index estimate for Laplace eigenfunctions claims

$$
\sup _{B_{g}(p, 2 r)}|\varphi| \leq 2^{C \sqrt{\lambda}} \sup _{B_{g}(p, r)}|\varphi|,
$$

where $C=C(M, g), p$ is any point on $W$ and $r \in\left(0, R_{0}(M, g)\right)$. It implies that the doubling index of $u$ is also bounded by $C_{1} \sqrt{\lambda}$ in balls with radius smaller than some $R_{1}=R_{1}(W, g)$. Let us fix a point $O \in M$ and a point $\widetilde{O}=(O, 0) \in M$. We can apply Theorem 6.1 to see that $H^{n}(\{u=0\} \cap$ $B(\widetilde{O}, r)) \leq C_{2} \lambda^{\alpha}$ for some $r=r(W, g)>0$.

It remains to note that $H^{n}(\{u=0\} \cap B(\widetilde{O}, r)) \leq C_{2} \lambda^{\alpha}$ implies $H^{n-1}(\{\varphi=$ $\left.0\} \cap B_{g}(O, r / 2)\right) \leq C_{3} \lambda^{\alpha}$ since the zero set of $u$ is exactly $Z_{\varphi} \times \mathbb{R}$. Finally, one can cover $M$ by finite number of such balls and obtain the desired global estimate of the volume of the nodal set.

Remark. The same argument gives a local volume estimate of the nodal set:

$$
H^{n-1}\left(\{\varphi=0\} \cap B_{g}(O, r)\right) \leq C r^{n-1} \lambda^{\alpha} .
$$

\section{Auxiliary LEMmas}

Lemma 7.1. If $\varepsilon_{1}>0$ is a sufficiently small number $\left(\varepsilon_{1}<1 / 10^{10}\right)$, then there exist $C=C\left(\varepsilon_{1}, M, g, O\right)>0$ and $R_{1}=R_{1}\left(\varepsilon_{1}, M, g, O\right)>0$ such that

$$
\beta\left(p, 2 r\left(1+\varepsilon_{1}\right)\right)\left(1+100 \varepsilon_{1}\right)+C \geq N(p, r) \geq \beta\left(p, r\left(1+\varepsilon_{1}\right)\right)\left(1-100 \varepsilon_{1}\right)-C
$$

for $r \in\left(0, R_{1}\right)$ and $p \in B\left(O, R_{1}\right)$.

We remark that it is not a misprint and the argument in $\beta$ in the righthand side of (15) is strictly greater than $r$.

Proof. By the equivalence of metrics (4) we have $B(p, r) \subset B_{g}(p, r(1+\varepsilon))$ and by the standard elliptic estimate

$$
\sup _{B(p, r)}|u|^{2} \leq \sup _{B_{g}(p, r(1+\varepsilon))}|u|^{2} \leq C_{1} H\left(r(1+\varepsilon)^{2}\right) / r^{n-1}
$$

and

$$
\sup _{B(p, 2 r)}|u|^{2} \geq \sup _{B_{g}(p, 2 r(1-\varepsilon))}|u|^{2} \geq C_{2} H(2 r(1-\varepsilon)) / r^{n-1} .
$$

Hence we can estimate

$$
N(p, r)=\frac{1}{2} \log _{2} \frac{\sup _{B(p, 2 r)}|u|^{2}}{\sup _{B(p, r)}|u|^{2}} \geq \frac{1}{2} \log _{2} \frac{1}{C_{3}} \frac{H(2 r(1-\varepsilon))}{H\left(r(1+\varepsilon)^{2}\right)},
$$


and by Corollary 1.2 the right-hand side is at least

$$
\log _{2}\left[\frac{1}{C_{3}}\left(\frac{2(1-\varepsilon)}{(1+\varepsilon)^{2}}\right)^{\beta\left(r(1+\varepsilon)^{2}\right) /(1+\varepsilon)}\right] \geq \beta\left(r(1+\varepsilon)^{2}\right)(1-20 \varepsilon)-C_{4} .
$$

We assumed above that $\varepsilon$ is sufficiently small. Now, we can let $\varepsilon_{1}$ be such that $(1+\varepsilon)^{2}=1+\varepsilon_{1}$, so $\varepsilon_{1} \sim 2 \varepsilon$, and the right-hand side inequality of (15) is obtained.

To obtain the opposite estimate we argue in the same manner:

$$
\sup _{B(p, r)}|u|^{2} \geq \sup _{B_{g}(p, r(1-\varepsilon))}|u|^{2} \geq C_{2} H(r(1-\varepsilon)) / r^{n-1},
$$

and

$$
\sup _{B(p, 2 r)}|u|^{2} \leq \sup _{B_{g}(p, 2 r(1+\varepsilon))}|u|^{2} \leq C_{3} H\left(2 r(1+\varepsilon)^{2}\right) / r^{n-1} .
$$

Applying these estimates we have

$$
N(p, r)=\log _{2} \frac{\sup _{B(p, 2 r)}|u|}{\sup _{B(p, r)}|u|} \leq \frac{1}{2} \log _{2} C_{4} \frac{H\left(2 r(1+\varepsilon)^{2}\right)}{H(r(1-\varepsilon))} .
$$

In a view of (1.2), the right hand side can be estimated from above by

$$
\beta\left(2 r(1+\varepsilon)^{2}\right)(1+20 \varepsilon)+C_{5} \leq \beta\left(p, 2 r\left(1+\varepsilon_{1}\right)\right)\left(1+100 \varepsilon_{1}\right)+C_{5},
$$

where $\varepsilon_{1}$ satisfies $(1+\varepsilon)^{2}=1+\varepsilon_{1}$.

Lemma 7.2. Let $\varepsilon$ be a small positive number. Then there exists $R=$ $R(\varepsilon, M, g, O)$ such that for any $x \in B(O, R)$ and for any numbers $t>2$ and $\rho>0$ such that $t \rho<R$,

$$
\sup _{B(x, t \rho)}|u| \geq t^{N(x, \rho)(1-\varepsilon)-C(\varepsilon, M, g)} \sup _{B(x, \rho)}|u| .
$$

Furthermore, there exists $N_{0}=N_{0}(\varepsilon, M, g)$ such that if $N(x, \rho)>N_{0}$, then additionally

$$
\sup _{B(x, t \rho)}|u| \geq t^{N(x, \rho)(1-\varepsilon)} \sup _{B(x, \rho)}|u| .
$$

Proof. We can assume that $t>2^{1+\varepsilon}$, otherwise $t^{N(x, \rho)(1-\varepsilon)} \leq 2^{N(x, \rho)}$ and

$$
\sup _{B(x, t \rho)}|u| \geq \sup _{B(x, 2 \rho)}|u| \geq 2^{N(x, \rho)} \sup _{B(x, \rho)}|u| \geq t^{N(x, \rho)(1-\varepsilon)} \sup _{B(x, \rho)}|u| .
$$

Hereafter the constants $C_{1}, C_{2}, \ldots$ will be positive numbers depending on $\varepsilon, M, g$ only. The inequality (3) says that

$$
\sup _{B(x, t \rho)}|u|^{2} \geq C_{6} \frac{H(x, t \rho)}{(t \rho)^{n-1}} .
$$

Let $\varepsilon_{1}$ be equal to $\varepsilon / 1000$. We can apply (15) for $\varepsilon_{1}$ to see that

$$
\beta\left(x, 2 \rho\left(1+\varepsilon_{1}\right)\right)\left(1+100 \varepsilon_{1}\right)+C_{7} \geq N(x, \rho) .
$$


In view of Corollary 1.2 we obtain

$$
H(x, t \rho) \geq H\left(x, 2 \rho\left(1+\varepsilon_{1}\right)\right)\left(\frac{t}{2\left(1+\varepsilon_{1}\right)}\right)^{\frac{2 N(x, \rho)}{\left(1+100 \varepsilon_{1}\right)\left(1+\varepsilon_{1}\right)}-C_{8}} .
$$

We use $t>2^{1+\varepsilon}$ to ensure that $t \rho>2 \rho\left(1+\varepsilon_{1}\right)$. A standard elliptic estimate yields

$$
H\left(x, 2 \rho\left(1+\varepsilon_{1}\right)\right) \geq C_{9} \rho^{n-1} \sup _{B(x, 2 \rho)}|u|^{2}=C_{9} 2^{2 N(x, \rho)} \rho^{n-1} \sup _{B(x, \rho)}|u|^{2} .
$$

Combination of (18), (19), (20) implies

$$
\begin{aligned}
\sup _{B(x, t \rho)}|u| & \geq C_{10} 2^{N(x, \rho)} t^{-(n-1) / 2}\left(\frac{t}{2\left(1+\varepsilon_{1}\right)}\right)^{N(x, \rho) /\left(1+200 \varepsilon_{1}\right)-C_{8} / 2} \sup _{B(x, \rho)}|u| \\
& \geq C_{10} t^{-(n-1) / 2}\left(\frac{t}{\left(1+\varepsilon_{1}\right)}\right)^{N(x, \rho) /\left(1+200 \varepsilon_{1}\right)-C_{11}} \sup _{B(x, \rho)}|u| .
\end{aligned}
$$

Now, in order to establish (16) it is sufficient to note that

$$
t^{N(x, \rho) /\left(1+200 \varepsilon_{1}\right)} \geq t^{N(x, \rho)(1-\varepsilon / 2)} \geq t^{N(x, \rho)(1-\varepsilon)} 2^{N(x, \rho) \varepsilon / 2}
$$

and

$$
2^{N(x, \rho) \varepsilon / 2} \geq\left(1+\varepsilon_{1}\right)^{N(x, p) /\left(1+200 \varepsilon_{1}\right)}
$$

since $2^{\varepsilon / 2} \geq 1+\varepsilon / 100$.

The inequality (17) follows immediately from (16) if we apply it to twice smaller $\varepsilon$, require $N(x, \rho)>\frac{2}{\varepsilon} C(\varepsilon / 2, M, g)$ and put a new smaller $R=$ $R(\varepsilon / 2, M, g)$.

Lemma 7.3. Let $\varepsilon$ be a small positive number. Then there exists $R=$ $R(\varepsilon, M, g, O)$ such that for any $x \in B(O, R)$ and any numbers $t>2$ and $\rho>0$ such that $t \rho<R$

$$
\sup _{B(x, t \rho)}|u| \leq t^{N(x, t \rho)(1+\varepsilon)+C(\varepsilon, M, g)} \sup _{B(x, \rho)}|u| .
$$

Furthermore, there exists $N_{0}=N_{0}(\varepsilon, M, g, O)$ such that if $N(x, \rho)>N_{0}$, then additionally

$$
\sup _{B(x, t \rho)}|u| \leq t^{N(x, t \rho)(1+\varepsilon)} \sup _{B(x, \rho)}|u| .
$$

Proof. The proof is parallel to the proof of the previous lemma. Put $\varepsilon_{1}=$ $\varepsilon / 1000$.

Inequality (2) says that

$$
\sup _{B(x, t \rho)}|u|^{2} \leq C_{1} \frac{H\left(x, t \rho\left(1+\varepsilon_{1}\right)\right)}{(t \rho)^{n-1}} .
$$

We can apply (15) for $\varepsilon_{1}$ to see that $\beta\left(x, t \rho\left(1+\varepsilon_{1}\right)\right) \leq N(x, t \rho)(1+$ $\left.100 \varepsilon_{1}\right)+C_{2}$. In view of the corollary $(1.2)$ we obtain

$$
H\left(x, t \rho\left(1+\varepsilon_{1}\right)\right) \leq H(x, \rho)\left(t\left(1+\varepsilon_{1}\right)\right)^{2 N(x, t \rho)\left(1+100 \varepsilon_{1}\right)\left(1+\varepsilon_{1}\right)+C_{3}} .
$$


Inequality (3) implies

$$
H(x, \rho) \leq C_{5} \sup _{B(x, \rho)}|u|^{2} \rho^{n-1} .
$$

Combination of (23), (24),$(25)$ gives us

$$
\begin{gathered}
\sup _{B(x, t \rho)}|u| \leq C_{6} \frac{\left(t\left(1+\varepsilon_{1}\right)\right)^{N(x, t \rho)\left(1+100 \varepsilon_{1}\right)\left(1+\varepsilon_{1}\right)+C_{3}}}{t^{(n-1) / 2}} \sup _{B(x, \rho)}|u| \\
\leq C_{7}\left(t\left(1+\varepsilon_{1}\right)\right)^{N(x, t \rho)\left(1+200 \varepsilon_{1}\right)+C_{3}} \sup _{B(x, \rho)}|u| .
\end{gathered}
$$

Noting that $t^{10 \varepsilon_{1}} \geq\left(1+\varepsilon_{1}\right)$, since $t>2$, we can estimate

$$
\left(t\left(1+\varepsilon_{1}\right)\right)^{N(x, t \rho)\left(1+200 \varepsilon_{1}\right)+C_{3}} \leq t^{N(x, t \rho)\left(1+500 \varepsilon_{1}\right)+C_{8}} .
$$

The proof of (21) is finished.

The inequality (22) follows immediately from (21) if we apply it to twice smaller $\varepsilon$, require $N(x, t \rho)>\frac{2}{\varepsilon} C(\varepsilon / 2, M, g)$ and put a new smaller $R=$ $R(\varepsilon / 2, M, g)$.

Lemma 7.4. There exist $r=r(M, g, O)$ and $N_{0}=N_{0}(M, g, O)$ such that for any points $x_{1}, x_{2} \in B(O, r)$ and $\rho$ such that $N\left(x_{1}, \rho\right)>N_{0}$ and $d\left(x_{1}, x_{2}\right)<\rho<r$, there exists $C=C(M, g, O)>0$ such that

$$
N\left(x_{2}, C \rho\right)>\frac{99}{100} N\left(x_{1}, \rho\right) .
$$

Proof. One can choose such numerical $C$ that $B\left(x_{2}, C \rho\right) \supset B\left(x_{1}, C \rho(1-\right.$ $\left.\left.1 / 10^{10}\right)\right)$ and $B\left(x_{2}, C \rho / 2\left(1-10^{-9}\right)\right) \subset B\left(x_{1}, C \rho\left(1-1 / 10^{10}\right)\right)$. It follows from (17) and (22) that if we choose $t$ and $N_{0}$ properly, then

$$
\begin{gathered}
2^{N\left(x_{2}, C \rho\right)(1+1 / 1000)} \geq \frac{\sup _{B\left(x_{2}, C \rho\right)}|u|}{\sup _{B\left(x_{2}, C \rho / 2\left(1-10^{-9}\right)\right)}|u|} \\
\geq \frac{\sup _{B\left(x_{1}, C \rho\left(1-10^{-10}\right)\right)}|u|}{\sup _{B\left(x_{1}, C \rho / 2\left(1-10^{-10}\right)\right)}|u|} \geq 2^{N\left(x_{1}, \rho\right)(1-1 / 1000)} .
\end{gathered}
$$

Thus

$$
N\left(x_{2}, C \rho\right)>\frac{999}{1001} N\left(x_{1}, \rho\right)>\frac{99}{100} N\left(x_{1}, \rho\right)
$$

\section{REFERENCES}

[1] G. Alessandrini, L. Rondi, E. Rosset and S. Vessella, The stability for the Cauchy problem for elliptic equations, Inverse Problems 25 (2009), no. 12, 123004, 47 pp.

[2] J.Brüning, Über Knoten von Eigenfunktionen des Laplace-Beltrami Operator, Math Z. 158 (1978), 15-21.

[3] T.H.Colding, W.P.Minicozzi II, Lower Bounds for Nodal Sets of Eigenfunctions, Comm. Math. Phys. 306 (2011), 777-784. 
[4] Dong, Rui-Tao. Nodal sets of eigenfunctions on Riemann surfaces. J. Differential Geom. 36 (1992), no. 2, 493-506. http://projecteuclid.org/euclid.jdg/1214448750.

[5] H. Donnelly, C. Feffermann, Nodal sets of eigenfunctions on Riemannian manifolds, Invent. Math., 93 (1988), 161-183.

[6] H. Donnelly, C. Feffermann, Nodal sets for eigenfunctions of the Laplacian on surfaces, J. Amer. Math. Soc., 3 (1990), 333-353.

[7] D.Gilbarg, N.S.Trudinger, Elliptic Partial Differential Equations of Second Order, Springer, 1998.

[8] N. Garofalo, F.-H. Lin, Monotonicity properties of variational integrals, $A_{p^{-}}$ weights and unique continuation, Indiana Univ. Math. J., 35 (1986), 245-268.

[9] R. Hardt and L. Simon, Nodal sets for solutions of elliptic equations. J. Differential Geom. 30 (1989), no. 2, 505-522.

[10] Q. Han, F.-H. Lin, Nodal Sets of Solutions of Elliptic Differential Equations, book in preparation.

[11] F.-H. Lin, Nodal sets of solutions of elliptic and parabolic equations, Comm. Pure Appl. Math., 44 (1991), 287-308.

[12] A.Logunov, Eu. Malinnikova, Nodal sets of Laplace eigenfunctions: estimates of the Hausdorff measure in dimension two and three, preprint.

[13] A.Logunov, Nodal sets of Laplace eigenfunctions: proof of Nadirashvili's conjecture and of the lower bound in Yau's conjecture, preprint.

[14] D. Mangoubi, The effect of curvature on convexity properties of harmonic functions and eigenfunctions, J. Lond. Math. Soc., 87 (2013), 645-662.

[15] N. Nadirashvilli, Geometry of nodal sets and multiplicity of eigenvalues, Current Developments in Mathematics, 1997, 231-235.

[16] F. Nazarov, L. Polterovich, M. Sodin, Sign and area in nodal geometry of Laplace eigenfunctions, Amer. J. Math., 127 (2005), 879-910.

[17] C.D.Sogge, S. Zelditch, Lower bounds on the Hausdorff measure of nodal sets II, Math. Res. Lett. 19 (2012), 1361-1364.

[18] S.-T. Yau, Problem section, Seminar on Differential Geometry, Annals of Mathematical Studies 102, Princeton, 1982, 669-706.

School of Mathematical Sciences, Tel Aviv University, Tel Aviv 69978, ISRAEL

Chebyshev Laboratory, St. Petersburg State University, 14th Line V.O., 29B, Saint Petersburg 199178 Russia

E-mail address: log239@yandex.ru 